\section{Audaces fortuna juvat}

Einstimmig haben die Delegierten der Ärztekammer an der ausserordentlichen Sitzung vom 13. Dezember 2001 dem Antrag des Zentralvorstands, die Urabstimmung zum gesamtschweizerischen Einheitstarif TARMED durchzuführen, zugestimmt. Damit sind die statutarischen Voraussetzungen erfüllt, die Durchführung der Urabstimmung ohne Verzug einzuleiten. Es sei hier daran erinnert, dass die Urabstimmung die Beschlussfassung aller stimmberechtigten Mitglieder der FMH auf schriftlichem Weg ist (vgl. www.fmh.ch, "Statuten»). Die Mitglieder werden folgende Abstimmungsfragen mit Ja oder Nein zu beantworten haben:

- Wollen Sie die Tarifstruktur TARMED 1.1 als Einführungsversion annehmen?

- Wollen Sie dem Rahmenvertrag UV/IV/MV zustimmen?

- Wollen Sie dem Rahmenvertrag KVG zustimmen?

Mit der Beantwortung dieser Fragen wird über die grundsätzliche Zustimmung der Ärzteschaft über die Einführung von TARMED entschieden.

Wie sieht nun der weitere Fahrplan aus? In einer der folgenden Ausgaben der Schweizerischen Ärztezeitung werden Sie das Protokoll der ausserordentlichen Ärztekammersitzung mit den ausführlichen Schilderungen der Verhandlungen und der Abstimmung nachlesen können. Als offizielle Bekanntgabe des Abstimmungsresultates gelten jedoch diese Zeilen. Den Stimmzettel mit den erforderlichen Unterlagen sowie den Erläuterungen zur Abstimmung er- halten alle FMH-Mitglieder Anfang Februar 2002 per Briefpost. Da die zur Abstimmung gelangenden Unterlagen äusserst umfangreich sind, werden sie auf einer CD-ROM verschickt. Wichtig für alle Mitglieder ohne Computer: Die Printversion der Version 1.1 erscheint als Supplementum zur Nummer 4 (23. Januar 2002) der Schweizerischen Ärztezeitung. Die weiteren relevanten Dokumente erscheinen in der Nummer 5 (30. Januar 2002) und im Supplementum zur selben Ausgabe der Schweizerischen Ärztezeitung.

Die Mitglieder haben rund einen Monat Zeit, den ausgefüllten Stimmzettel im Rückantwortcouvert zu retournieren. Das Ergebnis der Abstimmung wird Ende März erwartet. Für die Dauer der Urabstimmung wird eine Info-Hotline installiert, die allen FMH-Mitgliedern zur Verfügung steht. Wir werden die entsprechende Telefonnummer im voraus bekanntgeben.

Die Urabstimmung ist eine einmalige Gelegenheit, uns allen die demokratischen Prinzipien unserer ärztlichen Dachorganisation ins Bewusstsein zu rufen. Wie auch immer wir zu TARMED abstimmen werden, eines sollten wir uns nicht entgehen lassen: durch 100prozentige Stimmbeteiligung unseren Opponenten, unseren Partnern, aber auch uns selbst gegenüber Geschlossenheit und Standfestigkeit zu demonstrieren. Das scheint mir eine Frage der Selbstachtung zu sein.

Gesegnete Weihnachten und ein gutes Neues Jahr!

Dr. med. Markus Trutmann Chefredaktor Schweizerische Ärztezeitung 